\title{
Tratamiento de lodos generados en el proceso convencional de potabilización de agua
}

\author{
| Johnatan Alberto Gutiérrez-Rosero* \\ Álvaro Ignacio Ramírez-Fajardo** \\ Rodrigo Rivas ${ }^{* * *}$ \\ Balmes Linares"** \\ Diego Paredes
}

Recibido: 15/03/2014 - Aceptado: 27/06/2014

\begin{abstract}
Resumen
Se investigaron los lodos aluminosos del proceso de sedimentación de una planta convencional de potabilización de agua sometidos a ensayos de recuperación de aluminio, adicionando $\mathrm{H}_{2} \mathrm{SO}_{4}$, sedimentabilidad y espesamiento. Utilizando $\mathrm{H}_{2} \mathrm{SO}_{4}$, se recuperó el 60-70 \% de la concentración inicial del aluminio en el lodo, con $\mathrm{pH}=1,5$ Und. y mezcla de 5-30 minutos. Para los ensayos de sedimentabilidad y espesamiento se utilizaron los polímeros Nalclear 8173 y Trafloc 496. Se obtuvieron remociones significativas para SST y turbiedad en la sedimentabilidad con un nivel de significancia de 0,05 ; no hubo diferencias significativas entre los polímeros; la dosis que presentó eficiencias de remoción significativas fue $10 \mathrm{mg} / \mathrm{L}$. En el espesamiento, la adición de Trafloc y el factor tiempo influyeron significativamente sobre las eficiencias de remoción de DQO, DBO, SST y turbiedad; no hubo diferencias significativas entre las dosis de polímero utilizadas. La sedimentabilidad presentó mejores condiciones técnicas para el tratamiento de los lodos ensayados.
\end{abstract}

Palabras clave: lodos de PTAP, recuperación de aluminio, polímeros, sedimentabilidad, espesamiento.

\footnotetext{
Ing. Sanitario, Asistente de investigación, Facultad de Ciencias Ambientales, Universidad Tecnológica de Pereira, Colombia.johnguti@utp.edu.co

** Administrador Ambiental, Magister Ecotecnología, Profesor asistente, Facultad de Ciencias Ambientales, Universidad Tecnológica de Pereira, Colombia. aligrafa@utp.edu.co

*** Ing. Sanitario, Estudiante Maestría Ecotecnología, Grupo de Investigación Aguas \& Aguas de Pereira, Colombia. rarivas@aguasyaguas.com.co

**** Químico Industrial, Estudiante Maestría Ecotecnología, Grupo de Investigación Aguas \& Aguas de Pereira, Colombia.blinares@aguasyaguas.com.co

***** Ing. Sanitario, MSc. Dr. Profesor Titular, Facultad de Ciencias Ambientales, Universidad Tecnológica de Pereira, Colombia.diparede@utp.edu.co. Autor de correspondencia
} 


\title{
Treatment of waterworks sludge from conventional process to drinking water
}

\begin{abstract}
Waterworks sludge from sedimentation process in conventional drinking water treatment plant, recovery tests under aluminum adding $\mathrm{H}_{2} \mathrm{SO}_{4}$, thickening and settling properties were investigated. Using $\mathrm{H}_{2} \mathrm{SO}_{4}$, was recovered $60-70 \%$ of the initial concentration of aluminum in the sludge, and $\mathrm{pH}=1.5$ Und mixture 5-30 minutes. For testing the settleability and thickening Nalclear 8173 and Trafloc 496 polymers were used. With a significance level of 0.05 , the settling properties significant for SST and turbidity removal was obtained; there were no significant differences between the polymers; dose efficiencies presented significant removal was $10 \mathrm{mg} / \mathrm{L}$. In thickening, adding Trafloc and the time factor influenced significantly on removal efficiencies of COD, BOD, TSS and turbidity; there were no significant differences between the doses of polymer used. The settleability provided best technical conditions for the treatment of sludge tested.
\end{abstract}

Key words: waterworks sludge, aluminum recovery, polymers, settleability, thickening. 


\section{INTRODUCCIÓN}

En las plantas convencionales de potabilización de agua -PTAP- que incluyen coagulación, floculación, sedimentación y filtración, los lodos se producen en todos los procesos, excepto en la coagulación. Los coagulantes químicos más utilizados en sistemas de potabilización de agua son las sales de aluminio (i. e. sulfato y policloruro de aluminio) y las de hierro (i. e. cloruro y sulfato férrico) [1, 2]. Al respecto, Babatunde et al. [3] señalan que estas sales tienen potencial de reutilización y ofrecen excelentes posibilidades de comercialización.

Las propiedades de los lodos generados en las PTAP varían en el tiempo, incluso en un mismo sistema de tratamiento, debido principalmente a la calidad del agua afluente y a los regímenes de coagulación determinados en tal sistema $[4,5] \mathrm{y}$, aunque son considerados inertes [6], han sido tratados como residuo especial debido a que las sustancias presentes en el agua sin tratar tales como partículas en suspensión, arenas, arcillas, coloides, materia orgánica y microrganismos, son retenidas en los lodos [7].

El manejo que se le ha dado a los lodos efluentes de PTAP basados en coagulación química incluye el almacenamiento en lagunas por períodos de tiempo indefinidos, incineración, espesamiento y deshidratación [8]; en estas últimas se obtienen reducciones de humedad entre $30-40 \%$ para llevarlos a disposición final $[9,10]$. No obstante, debido a que sus propiedades mecánicas incrementan proporcionalmente con las dosis de coagulante utilizado, estas operaciones pueden resultar complejas y, en la mayoría de los casos, su disposición en fuentes de agua superficial resulta ser la alternativa de manejo preferida [11] debido a lo simple de esta operación en las plantas, a la carencia de terrenos cercanos para su disposición y a los altos costos de las tecnologías convencionales para su tratamiento, con lo cual se generan problemas para el ambiente y la salud humana [1,12]. Li et al. [13] señalan que el drenaje de lodos de las unidades sedimentadoras representa el 1,5-2,0 \% del volumen total del agua generada en las PTAP. Factores como la construcción y ampliación de las PTAP inciden directamente en la producción mundial de lodos que, para el año 1997, superaba las 10 mil t/día [14], haciendo que la implementación de las unidades de tratamiento sea limitada debido a sus altos costos de construcción, mantenimiento y operación [10] razones por las cuales el manejo y disposición final de estos lodos resulta ser un desafío.

En Colombia, la información publicada en torno a experiencias de reutilización y tratamiento de los lodos provenientes de PTAP es escasa. En tres PTAP de ciclo convencional de diferentes ciudades del país, se realiza el tratamiento de estos lodos donde prima el tratamiento físico por medio de la deshidratación, mientras que en el municipio de Caldas (Antioquia) se cuenta con una planta piloto para el tratamiento de lodos donde se espesan por medio de polímeros hasta su secado para su posterior 
disposición. Asimismo, han sido pocas las publicaciones para la reutilización y tratamiento de lodos provenientes de PTAP [1,15].

La recuperación del aluminio contenido en los lodos de plantas potabilizadoras por medio de la acidificación del medio es otra de las alternativas para el aprovechamiento de estos residuos. Se han reportado porcentajes de recuperación de aluminio mayores al $90 \%$ en lodos provenientes de PTAP, que se han utilizado en el tratamiento primario avanzado de aguas residuales domésticas alcanzando remociones de materia orgánica en DQO superiores al $85 \%[16,17]$.

Entre los métodos más utilizados para el tratamiento de los lodos generados en los sistemas convencionales de potabilización de agua se encuentra la sedimentación con la adición de polímeros floculantes $[4,6,18]$. En aguas con altas turbiedades $(>1000$ UNT), como el drenaje de lodos, la adición de $0,1 \mathrm{mg} / \mathrm{L}$ de polímero puede alcanzar remociones de hasta $50 \%$ cuando estos se incorporan a procesos de sedimentabilidad, previa mezcla rápida $[6,19]$.

El espesamiento y la deshidratación son tecnologías que reducen los costos de manejo y transporte de lodos obtenidos en las PTAP, y la adición de polímeros sintéticos ha sido probada eficientemente para estos fines, incrementando la densidad de los flocs y la velocidad de sedimentación [9,19]. En esta investigación se utilizaron lodos provenientes de las unidades de sedimentación de una PTAP con tratamiento convencional en una ciudad del Eje Cafetero colombiano para determinar su toxicidad en términos fisicoquímicos y ser sometidos a procesos de recuperación de aluminio en medio ácido, y de sedimentabilidad y espesamiento con polímeros ayudantes de coagulación, con el fin de identificar la tecnología más eficiente técnicamente para el tratamiento de los lodos producidos en el proceso de potabilización de agua.

\section{MATERIALES Y MÉTODOS}

El estudio se desarrolló en las instalaciones de la PTAP de Aguas \& Aguas de la ciudad de Pereira en el Eje Cafetero colombiano, que realiza tratamiento convencional y se abastece de una fuente de agua superficial para la cual utilizan sulfato de aluminio $\mathrm{Al}_{2}\left(\mathrm{SO}_{4}\right)_{3}-$ en el proceso de coagulación. Los lodos de las unidades de tratamiento de la PTAP como desarenador, floculador, sedimentador y baterías de filtros se caracterizaron en términos de DQO, DBO, ST, SST, SVT, SS para las temporadas climáticas predominantes en la zona, temporada lluviosa y seca, las cuales se presentaron entre los meses de septiembre-noviembre y diciembre-febrero, respectivamente. Con base en estos resultados y los reportes de la literatura, se determinó que los lodos objetos de estudio serían los efluentes de los sedimentadores, a los cuales se les efectuaron análisis de lixiviación característica de toxicidad (En inglés TCLP). Se evaluaron tres 
alternativas para su aprovechamiento y tratamiento: i) Recuperación del aluminio contenido en los lodos por medio ácido ii) Sedimentabilidad y iii) Espesamiento con adición de polímeros.

\subsection{Recuperación de aluminio de lodo en medio ácido}

Para la recuperación del aluminio residual presente en los lodos aluminosos, se siguió la metodología desarrollada por diversos autores $[15,17]$. Se realizaron ensayos preliminares para determinar el porcentaje de recuperación de aluminio a 5, 30 y 100 minutos con valores de $\mathrm{pH}$ entre 1,5 - 3,5 Und. A partir de lo anterior, los experimentos se enfocaron en ensayos con tiempos de 5 y 30 minutos y valores de $\mathrm{pH}$ de 1,5 Und. y consistieron en la homogeneización de la muestra de lodo de purga de los sedimentadores mediante mezcla rápida de 300 RPM durante 1 min en equipos Jart Test, mientras se adicionaba $\mathrm{H}_{2} \mathrm{SO}_{4} .98 \%$ para ajustar el $\mathrm{pH}$ de la mezcla a 1,5 Und.

Luego se disminuyó la velocidad de la mezcla a 100 RPM durante 30 minutos, tiempo durante el cual se recolectaron muestras de sobrenadante a los 5 y 30 minutos. Estas muestras sedimentaron durante $24 \mathrm{~h}$ más, al cabo de las cuales todas fueron filtradas y de sus sedimentos se tomaron otras muestras. Los análisis de aluminio a las muestras recolectadas se realizaron por el método de absorción atómica.

\subsection{Sedimentabilidad}

Se recolectaron muestras del drenaje de las unidades sedimentadoras de la PTAP objeto de estudio y se utilizaron dos polímeros comerciales, Nalclear 8173 y Trafloc 496 uno catiónico y otro no aniónico, respectivamente. El montaje constaba de una columna de sedimentación como la mostrada en la figura 1a. Cada ensayo se realizó con $12 \mathrm{~L}$ de muestra a 200 RPM durante un minuto y luego una hora de sedimentación. Al finalizar el tiempo de sedimentación, se tomaron muestras del sobrenadante de cada uno de los ensayos para la medición de las variables de respuesta DBO, DQO, SST y turbiedad. Los tratamientos utilizados en el ensayo de sedimentabilidad se presentan en la tabla 1. Los datos obtenidos fueron sometidos a pruebas estadísticas de normalidad de Shapiro - Wilks y de análisis de varianzas no paramétricas como la Kruskal Wallis para verificar diferencias entre los grupos formados por los factores Dosis (5, $10,15,20,25,30 \mathrm{mg} / \mathrm{L}$ ) y Polímero (Nalclear, Trafloc) para las variables REMDQO - Remoción de DQO, REMDBO - Remoción de DBO, REMSST - Remoción de SST y REMTURB - Remoción de Turbiedad para un nivel de significancia de 0,05 $(\alpha=0,05)$ mediante el paquete estadístico Statistical Package Social Science - SPSS v. $14.0\left(\mathrm{IBM}^{\circledR}\right)$. 


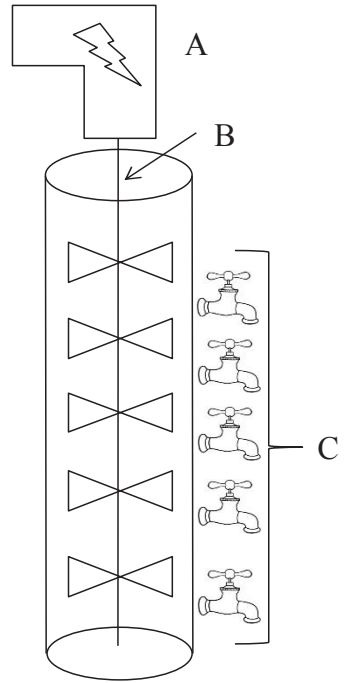

(a)

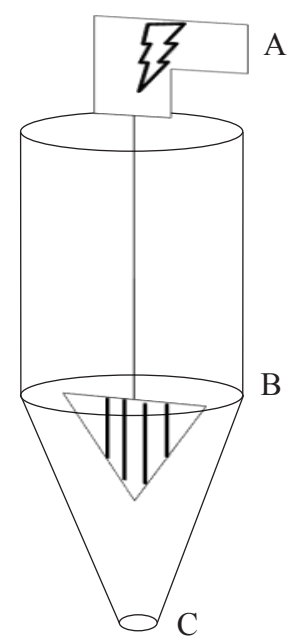

(b)

Figura 1. Esquema de montaje para ensayos de sedimentabilidad (a) y espesamiento (b) A: Motomezclador. B: Eje mezclador. C:Toma de muestras Fuente: Ios autores

Tabla 1. Tratamientos de los ensayos de sedimentabilidad y espesamiento de lodos

\begin{tabular}{|c|c|c|}
\hline \multirow{2}{*}{ Dosis $(\mathrm{mg} / \mathrm{L})$} & \multicolumn{2}{|c|}{ Polimero } \\
\cline { 2 - 3 } & Nalclear & Trafloc \\
\hline \multicolumn{3}{|c|}{ Sedimentabilidad } \\
\hline 0 & $\mathrm{~S} 1$ & $\mathrm{~S} 2$ \\
\hline 5 & $\mathrm{~S} 3$ & $\mathrm{~S} 4$ \\
\hline 10 & $\mathrm{~S} 5$ & $\mathrm{~S} 6$ \\
\hline 15 & $\mathrm{~S} 7$ & $\mathrm{~S} 8$ \\
\hline 20 & $\mathrm{~S} 9$ & $\mathrm{~S} 10$ \\
\hline 25 & $\mathrm{~S} 11$ & $\mathrm{~S} 12$ \\
\hline 30 & $\mathrm{~S} 13$ & $\mathrm{~S} 14$ \\
\hline
\end{tabular}

\begin{tabular}{|c|c|c|}
\hline \multicolumn{3}{|c|}{ Espesamiento } \\
\hline 0 & E1 & E2 \\
\hline 10 & E3 & E4 \\
\hline 15 & E5 & E6 \\
\hline 20 & E7 & E8 \\
\hline
\end{tabular}

Fuente: los autores 


\subsection{Espesamiento}

Los ensayos de espesamiento se realizaron con lodos del fondo de los sedimentadores, utilizando un espesador de forma cónica provisto de una paleta mezcladora unida a un moto-reductor al cual se le adicionaban $12 \mathrm{~L}$ de muestra para cada ensayo (ver figura 1b). Los tiempos de espesamiento fueron 2 y 4 horas, y la velocidad de giro del motor fue 5 RPM. Al final de cada ensayo se tomaron muestras del sobrenadante y se midió el volumen de la fase sólida (lodo). Cada uno de los ensayos se realizó por triplicado. Los tratamientos evaluados se presentan en la tabla 1. Los datos obtenidos al final del experimento se procesaron mediante el paquete estadístico SPSS v. $14.0\left(\right.$ IBM $\left.^{\circledR}\right)$ realizando las mismas pruebas utilizadas en el ensayo de sedimentabilidad.

\section{RESULTADOS Y DISCUSIÓN}

\subsection{Caracterización de lodos efluentes de planta de potabilización}

En la tabla 2 se presentan los resultados de dos muestreos realizados a cada una de las unidades de tratamiento en la PTAP en las cuales se producen lodos. La mayor cantidad de sólidos se presentó en los lodos provenientes de las unidades de sedimentación, los que, además, presentan mayores concentraciones en los otros parámetros de interés ambiental como DQO. No obstante, los resultados en términos de DBO son similares en comparación con los lodos provenientes de otras unidades. Lo anterior corrobora lo encontrado por Torres et al. [1] y Li et al. [13], quienes indican que la mayor cantidad de sólidos se acumula en la unidad de sedimentación; en las demás unidades, aunque hay presencia de sedimentos, tales cantidades se pueden considerar despreciables comparadas con las producidas en el sedimentador. En la tabla 3 se presentan los resultados de la caracterización en términos de TCLP, que se encuentran muy por debajo de los límites establecidos como máximos, indicando que los lodos no presentan características fisicoquímicas para ser considerados como residuos peligrosos de acuerdo con los límites permisibles establecidos en el Decreto 4741/2005 del Ministerio de Ambiente, Vivienda y Desarrollo Territorial -MAVDT-. Lo anterior también coincide con los bioensayos realizados por Sotero-Santos et al. [20], quienes evaluaron la toxicidad de lodos de PTAP utilizando Daphnia similis. 
20 J. A. Gutiérrez-Rosero • Á. I. Ramírez-Fajardo • Rodrigo Rivas • B. Linares • D. Paredes

Tabla 2. Resultados caracterización de lodos efluentes de la planta de potabilización.

\begin{tabular}{|l|c|c|c|c|c|c|c|c|}
\hline \multirow{2}{*}{ Parámetro } & \multicolumn{2}{|c|}{ Desarenador } & \multicolumn{2}{c|}{ Floculador } & \multicolumn{2}{c|}{ Sedimentador } & \multicolumn{2}{c|}{ Filtros } \\
\cline { 2 - 10 } & $L$ & $S$ & $L$ & $S$ & $L$ & $S$ & $L$ & $S$ \\
\hline DQO $\left(\mathrm{mg} \mathrm{O}_{2} / \mathrm{L}\right)$ & 25,13 & 10,7 & - & 19,3 & 745 & 537 & 61,5 & 94 \\
\hline DBO $\left(\mathrm{mg} \mathrm{O}_{2} / \mathrm{L}\right)$ & 1,2 & 1,3 & - & 0,01 & 1,21 & 2,9 & 1,2 & 6,3 \\
\hline ST $(\mathrm{mg} / \mathrm{L})$ & 103 & 177 & - & 151 & 3098 & 930 & 209 & 334 \\
\hline SST $(\mathrm{mg} / \mathrm{L})$ & 16 & 26 & - & 30 & 2720 & - & 89 & 173 \\
\hline SVT $(\mathrm{mg} / \mathrm{L})$ & 33 & 53 & - & 59 & 1444 & 509 & 61 & 123 \\
\hline SSed $(\mathrm{mL} / \mathrm{L})$ & 0,1 & 0,01 & - & 0,01 & 245 & 60 & 0,4 & 4 \\
\hline
\end{tabular}

L: temporada de lluvias; S: temporada seca.

Fuente: los autores

Tabla 3. Resultados análisis TCLP de lodos de sedimentadores en dos temporadas climáticas

\begin{tabular}{|c|c|c|c|c|c|c|}
\hline Parámetro & Unds. & Técnica Analítica & Método & $\mathrm{L}$ & S & Límites* \\
\hline $\mathrm{pH}$ & Unds. & Electrométrico & $\mathrm{SM} 4500 \mathrm{H}+\mathrm{B}$ & 5,3 & 5,96 & N.E. \\
\hline Fenoles Totales & $\mathrm{mg} / \mathrm{L}$ & $\begin{array}{l}\text { Cromatografía de } \\
\text { gases - Masas }\end{array}$ & EPA 8041 & $<0,0010$ & $<0,0010$ & N.E. \\
\hline Bario & $\mathrm{mg} / \mathrm{L}$ & E.A.A. & SM 3030 E - SM 3111 D & 1,73 & 0,258 & $<100$ \\
\hline Cadmio & $\mathrm{mg} / \mathrm{L}$ & E.A.A. & SM 3030 E - SM 3111 B & $<0,007$ & $<0,009$ & $<1,0$ \\
\hline Cromo Total & $\mathrm{mg} / \mathrm{L}$ & E.A.A. & SM 3030 E - SM 3111 D & 0,2 & $<0,057$ & $<5,0$ \\
\hline Mercurio & $\mathrm{mg} / \mathrm{L}$ & E.A.A./V.F. & SM 3112 B & 0,17 & $<0,10$ & $<0,2$ \\
\hline Plata & $\mathrm{mg} / \mathrm{L}$ & E.A.A. & SM 3030 E - SM 3111 B & $<0,002$ & $<0,062$ & $<5,0$ \\
\hline Arsénico & $\mathrm{mg} / \mathrm{L}$ & E.A.A.E & SM 3030 E - SM 3113 B & 0,0003 & 0,0003 & $<5,0$ \\
\hline Selenio & $\mathrm{mg} / \mathrm{L}$ & E.A.A.E & SM 3030 E - SM 3113 B & 0,00015 & 0,00015 & $<1,0$ \\
\hline Plomo & $\mathrm{mg} / \mathrm{L}$ & E.A.A. & SM 3030 E - SM 3111 B & $<0,05$ & $<0,061$ & $<5,0$ \\
\hline COV`s & $\mathrm{mg} / \mathrm{L}$ & $\begin{array}{l}\text { Cromatografía de } \\
\text { gases - FID }\end{array}$ & EPA 5020 - EPA 624 & $<0,012$ & $<0,0024$ & N.E. \\
\hline $\begin{array}{l}\text { Pesticidas } \\
\text { órgano-clorados }\end{array}$ & $\mathrm{mg} / \mathrm{L}$ & $\begin{array}{l}\text { Cromatografía de } \\
\text { gases - ECD }\end{array}$ & $\begin{array}{l}\text { EPA 8081A Y B - SM } \\
6630 \text { B Y C }\end{array}$ & $<0,0004$ & $<0,050$ & N.E. \\
\hline
\end{tabular}

E. A. A.: Espectrofotometría de absorción atómica.

E. A. A. E.: Espectrofotometría de absorción atómica electrotémica.

E. A. A. /V. F.: Espectrofotometría de absorción atómica/vapor frío.

* Permisibles establecidos por MAVDT mediante Decreto 4741 del 2005.

Fuente: los autores 


\subsection{Recuperación de aluminio por vía ácida}

En la figura 2 se presentan los resultados obtenidos para las dos pruebas preliminares de recuperación de aluminio residual de los lodos de purga, donde las mayores concentraciones de aluminio se recuperaron cuando el $\mathrm{pH}$ fue 1,5 Und. y los valores reportados para los ensayos de 100 minutos no fueron relevantes comparados con los tiempos de 5 y 30 minutos. A partir de estas pruebas se determinó que los ensayos se realizarían a 1,5 Und. de $\mathrm{pH}$ y a tiempos de 5 y 30 minutos. Asimismo, se presentan las concentraciones de aluminio medidas en el sobrenadante en los tres ensayos completos realizados, en los cuales se evidencia que la presencia de aluminio en dicha solución es inversamente proporcional al $\mathrm{pH}$, encontrándose que con los valores de $\mathrm{pH}$ evaluados en 1,5 Und., se recuperó la mayor cantidad de aluminio, es decir, que existe una relación de proporcionalidad entre el volumen de ácido adicionado y el aluminio recuperado.

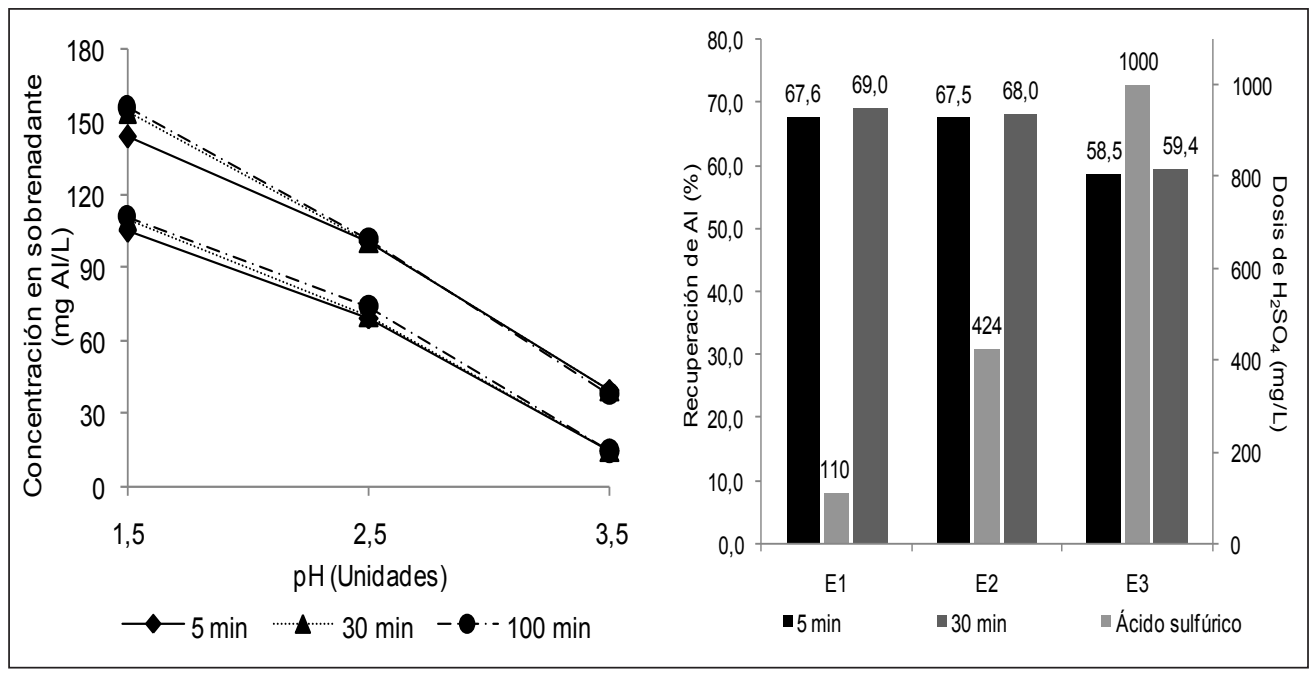

Figura 2. Concentraciones en ensayos preliminares con porcentajes de recuperación de aluminio del sobrenadante Fuente: los autores

Entre los tiempos de contacto en la mezcla no se presentan diferencias relevantes entre 5 y 30 minutos, corroborando los resultados de los ensayos preliminares. Lo anterior discrepa con lo encontrado por Azim et al. [17] donde los mayores porcentajes de recuperación de aluminio se alcanzaron a pH de 1,5 Und., pero entre 30-40 minutos de mezcla. Asimismo, estos resultados son similares a lo reportado por Villegas et al. [15] respecto de la relación entre la concentración de aluminio en el sobrenadante y los valores bajos de $\mathrm{pH}$ de la mezcla y los porcentajes de recuperación alcanzados cercanos a $70 \%$; sin embargo, difieren en los tiempos de mezcla, ya que en la actual 
investigación, estos no incidieron significativamente sobre los porcentajes de recuperación de aluminio (figura 2). No obstante, se requiere mayor evidencia experimental al respecto. Esta diferencia significativa de tiempo se traduce en menores costos de operación debido a que los requerimientos de electricidad para agitación serían menores.

\subsection{Sedimentabilidad}

El factor dosis arrojó diferencias significativas para un nivel de significancia de 0,05 $(\alpha=0,05)$ para la variable REMSST, mientras que en el factor Polímero se observaron diferencias significativas para las variables REMSST y REMTURB. En promedio, las eficiencias de remoción de SST y Turbiedad fueron 68 y $90 \%$, respectivamente. No se observaron diferencias significativas para las variables REMDQO y REMDBO.

En la tabla 4 se presentan las comparaciones entre las dosis utilizadas en los ensayos de sedimentabilidad. De acuerdo con los niveles de significancia solo existe diferencia relevante entre los grupos de dosis S5-S6 y S1-S2 $(0,024<0,05)$ respecto de la variable REMSST y por lo tanto, las diferencias entre los demás grupos de dosis no resultaron determinantes.

Tabla 4. Comparaciones agrupadas por parejas de factor DOSIS prueba de sedimentabilidad

\begin{tabular}{|c|c|c|c|c|c|c|}
\hline $\begin{array}{c}\text { Dosis 1 } \\
(\mathrm{mg} / \mathrm{L})\end{array}$ & Dosis 2 $(\mathrm{mg} / \mathrm{L})$ & $\begin{array}{c}\text { Prueba } \\
\text { estadistica }\end{array}$ & Error tipico & $\begin{array}{c}\text { Desv. Prueba } \\
\text { estadistica }\end{array}$ & Sig. & Sig. Ady. \\
\hline \multirow{5}{*}{ Blanco } & 30 & $-19,625$ & 9,269 & $-2,117$ & 0,034 & 0,719 \\
\cline { 2 - 7 } & 5 & $-20,500$ & 9,269 & $-2,112$ & 0,027 & 0,567 \\
\cline { 2 - 7 } & 25 & $-21,875$ & 9,269 & $-2,360$ & 0,018 & 0,384 \\
\cline { 2 - 7 } & 20 & $-26,562$ & 9,269 & $-2,866$ & 0,004 & 0,087 \\
\cline { 2 - 7 } & 15 & $-27,562$ & 9,269 & $-2,974$ & 0,003 & 0,062 \\
\hline \multirow{5}{*}{30} & 10 & $-30,125$ & 9,269 & $-3,250$ & 0,001 & 0,024 \\
\cline { 2 - 7 } & 25 & 0,875 & 7,568 & 0,116 & 0,908 & 1,000 \\
\cline { 2 - 7 } & 20 & 2,250 & 7,568 & 0,297 & 0,766 & 1,000 \\
\cline { 2 - 7 } & 15 & 7,938 & 7,568 & 0,917 & 0,359 & 1,000 \\
\hline \multirow{5}{*}{5} & 10 & 10,500 & 7,568 & 1,387 & 0,165 & 1,000 \\
\hline & 25 & $-1,375$ & 7,568 & $-0,182$ & 0,856 & 1,000 \\
\cline { 2 - 7 } & 20 & $-6,062$ & 7,568 & $-0,801$ & 0,423 & 1,000 \\
\cline { 2 - 7 } & 15 & $-7,062$ & 7,568 & $-0,933$ & 0,351 & 1,000 \\
\cline { 2 - 7 } & 10 & $-9,625$ & 7,568 & $-1,272$ & 0,203 & 1,000 \\
\hline
\end{tabular}




\begin{tabular}{|c|c|c|c|c|c|c|}
\hline \multirow{2}{*}{$\begin{array}{c}\text { Dosis 1 } \\
(\mathrm{mg} / \mathrm{L})\end{array}$} & Dosis 2 $(\mathrm{mg} / \mathrm{L})$ & $\begin{array}{c}\text { Prueba } \\
\text { estadistica }\end{array}$ & Error típico & $\begin{array}{c}\text { Desv. Prueba } \\
\text { estadistica }\end{array}$ & Sig. & Sig. Ady. \\
\hline \multirow{3}{*}{25} & 20 & 4,688 & 7,568 & 0,619 & 0,536 & 1,000 \\
\cline { 2 - 7 } & 15 & 5,688 & 7,568 & 0,752 & 0,452 & 1,000 \\
\cline { 2 - 7 } & 10 & 8,250 & 7,568 & 1,090 & 0,276 & 1,000 \\
\hline \multirow{2}{*}{20} & 15 & 1,000 & 7,568 & 0,132 & 0,895 & 1,000 \\
\hline \multirow{2}{*}{15} & 10 & 3,562 & 7,568 & 0,471 & 0,638 & 1,000 \\
\hline
\end{tabular}

Fuente: los autores

Con base en el resultado del ANOVA no paramétrico para el factor polímero se mostró que la significancia estaba en los grupos formados por las variables REMSST y REMTURB; en este sentido, en la figura 3 se encontró que, para un $\alpha=0,05$, las diferencias significativas ocurren en los tratamientos S1 y S2. Sin embargo, no hay diferencia entre los dos polímeros para las variables evaluadas.

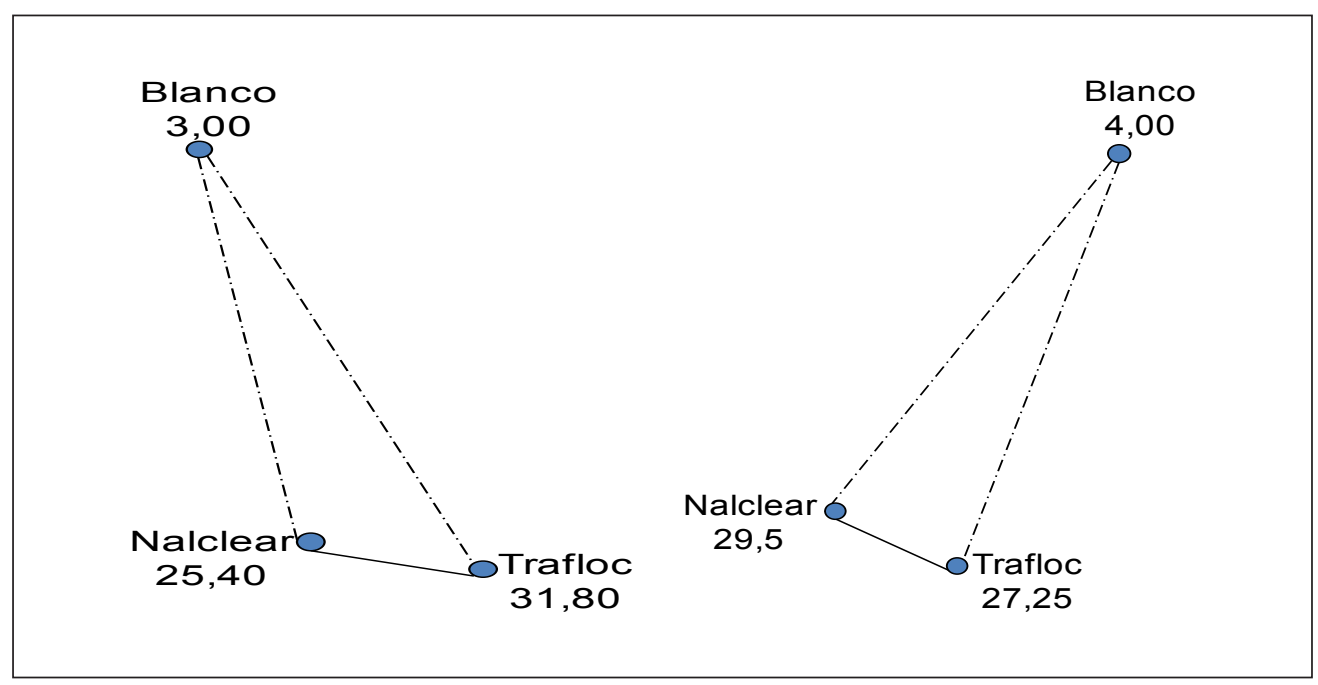

Figura 3. Comparaciones por parejas

del factor Polímero para variables REMSST y REMTURB en sedimentabilidad

Fuente: los autores

\subsection{Espesamiento}

Al igual que en los ensayos de sedimentabilidad, en el espesamiento los datos de las variables dependientes REMDQO, REMDBO, REMSST y REMTURB no obedecieron al supuesto de normalidad para un $\alpha=0,05$, por lo que fue necesario analizar los datos 
mediante la prueba Kruskal-Wallis para los factores i) Dosis, ii) Polímero y iii) Tiempo a 60 y 120 minutos; estos factores se analizaron para cada una de las variables dependientes. La prueba de hipótesis del análisis de varianza no paramétrico para el factor dosis mostró que es significativo en todas las variables, excepto en la REMDBO; además, se encontró que en los tratamientos E3 hasta E8 no existen diferencias significativas, razón por la cual es factible utilizar las dosis menores como E3 y E4 obteniéndose resultados estadísticamente iguales a los obtenidos con las dosis altas. Por otro lado, el factor Polímero ejerció un efecto significativo sobre las cuatro variables dependientes, presentando diferencias significativas en el tratamiento E2 para las variables REMDQO y REMDBO, mientras que para las variables REMSST y REMTURB los tratamientos E1 y E2 presentaron diferencias significativas en comparación con el Blanco; no hubo diferencias entre las comparaciones de ambos polímeros. Los resultados de estos análisis se presentan en la figura 4 , en donde las diferencias significativas por parejas en comparación con las muestras Blanco se representan mediante líneas punteadas.

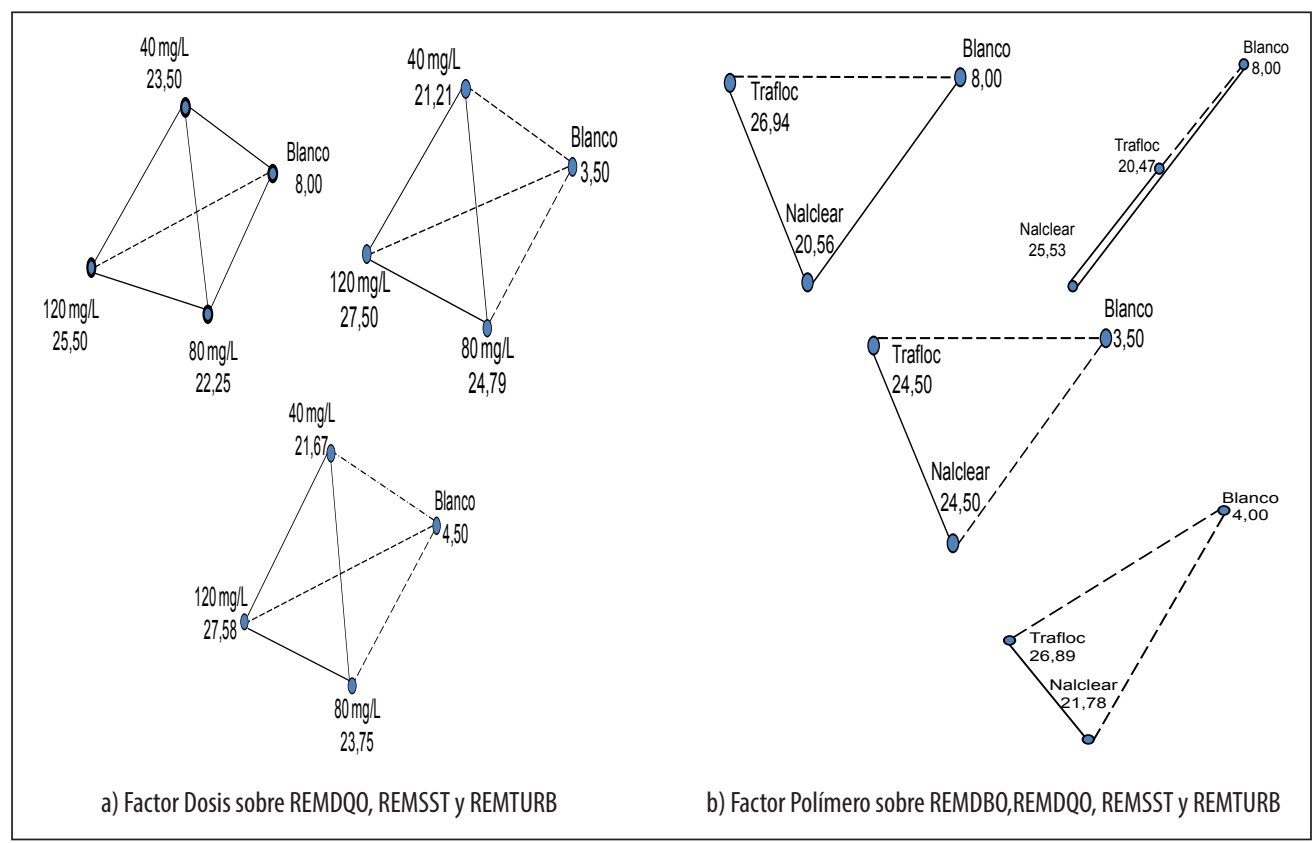

Figura 4. Comparaciones para parejas de los factoresDosis y Polímero para variables REMDBO,REMDQO, REMSST y REMTURB en espesamiento por gravedad.

Fuente: los autores

Respecto del factor tiempo, se realizó la prueba de hipótesis para verificar su influencia sobre las variables evaluadas, en la cual se encontró que, con un $\alpha=0,05$ este factor sí ejerce un efecto significativo; lo anterior significa que existe una relación 
directa de proporcionalidad entre el tiempo de los ensayos y las eficiencias de remoción de los parámetros evaluados, por lo cual las mayores eficiencias se alcanzaron a los 120 minutos.

\section{CONCLUSIONES}

Se determinó que el mayor porcentaje de lodos del sistema de potabilización estudiado proviene de las unidades de sedimentación. En términos fisicoquímicos y de acuerdo con las caracterizaciones realizadas, los lodos provenientes de estas unidades no se consideran un residuo peligroso, de acuerdo con la normativa vigente en el país. La recuperación de la mayor cantidad de aluminio en el sobrenadante se logró a valores de $\mathrm{pH}$ de 1,5 Unds., sin encontrar diferencias relevantes entre los tiempos de mezcla de 5 y 30 minutos. Mediante los experimentos de sedimentabilidad, se determinó, con un nivel de confianza del $95 \%$, que no existen diferencias significativas entre los dos polímeros utilizados en términos de remoción de SST y Turbiedad. Por otro lado, los tratamientos que presentaron un comportamiento significativo en términos de remoción de SST en comparación con los blancos y las demás dosis fueron S5 y S6. Las dosis ensayadas en el espesamiento no tuvieron diferencias significativas entre sí, pero incidieron significativamente sobre las eficiencias de remoción de las variables DQO, SST y turbiedad por lo que es factible utilizar dosis de polímero bajas obteniendo resultados estadísticamente iguales a los alcanzados con las altas; el tiempo fue directamente proporcional a las eficiencias de remoción de las variables DQO, SST y turbiedad. Asimismo, el polímero que presentó mayores diferencias significativas respecto del Blanco fue el Trafloc, lo que incide significativamente sobre los porcentajes de remoción de las variables dependientes evaluadas.

De acuerdo con los resultados de las tecnologías de tratamiento de lodos ensayadas, la sedimentabilidad es la que técnicamente presenta mayores ventajas debido a que utiliza dosis de polímero bajas y requiere tiempos más bajos para alcanzar eficiencias de remoción adecuadas, comparándola con la tecnología del espesamiento. Aunque se obtuvieron altos porcentajes de recuperación de aluminio por la vía ácida, esta tecnología se descarta debido a que requiere altas cantidades de $\mathrm{H}_{2} \mathrm{SO}_{4}$.

\section{AGRADECIMIENTOS}

Este trabajo fue financiado en el marco del Convenio Específico 017-2012 entre la Empresa de Acueducto y Alcantarillado de Pereira -Aguas y Aguas de Pereira- y la Universidad Tecnológica de Pereira. Los autores agradecen a la empresa Aguas y Aguas por facilitar sus instalaciones y al químico industrial Cristhian Alfredo Galeano por brindar el apoyo logístico necesario para la realización del trabajo reportado. 


\section{REFERENCIAS}

[1] P. Torres, et al., "Uso productivo de lodos de plantas de tratamiento de agua potable en la fabricación de ladrillos cerámicos". Revista ingeniería de construcción. Vol. 27, n. 3, pp. 145-154, 2012.

[2] S. Nielsen y D. J. Cooper. "Dewatering sludge originating in water treatment works in reed bed systems". Water Science and Technology. Vol. 64, n. ${ }^{\circ}$ 2, pp. 361-366, 2011.

[3] A. O. Babatunde, et al., "Characterization of aluminium, based water treatment residual for potential phosphorus removal in engineered wetlands". Environmental. Pollution. Vol. 157, n. ${ }^{\circ} 10$, pp. 2830-2836, 2009.

[4] Z. Gang, et al., "Full scale evaluation on ferric flocs sludge treatment with pelleting flocculation blanket process", presentado en Proceedings of the 2010 International Conference on Challenges in Environmental Science and Computer Engineering - CESCE. Wuhan, China, 2010.

[5] L. Spinosa. "Characterization: a necessary tool in sludge management". Water Science and Technology. Vol. 68, n. ${ }^{\circ}$ 4, pp. 748-755, 2013.

[6] D. I. Verrelli, et al., "Assessing dewatering performance of drinking water treatment sludges". Water Research, vol. 44, n. ${ }^{\circ}$ 5, pp. 1542-1552, 2010.

[7] W.-P. Cheng, et al., "Dynamics of aluminum leaching from water purification sludge". Journal of Hazardous Materials. Vol. 217, pp. 149-155, 2012.

[8] B. C. O'kelly. "Landfill disposal of alum water treatment residues: some pertinent geo-engineering properties". Journal of Residuals Science and Technology. Vol. 7, n. ${ }^{\circ}$, pp. 95-113, 2010.

[9] Y. Q. Zhao, "Correlations between floc physical properties and optimum polymer dosage in alum sludge conditioning and dewatering", Chemical Engineering Journal, vol. 92, n. ${ }^{\circ}$, pp. 227-235, 2003.

[10] S. S. Ferreira-Filho, et al., "Water treatment plant sludge disposal into stabilization ponds". Water Science and Technology, vol. 67, n. ${ }^{\circ}$ 5, pp. 1017-1025, 2013.

[11] M. E. Walsh, et al., "Strategic pathways for the sustainable management of wáter treatment plant residuals", Journal of Environmental Engineering and Science, vol. 7, n. ${ }^{\circ}$, pp. 45-52, 2008 .

[12] A. N. Victoria, "Characterisation and performance evaluation of waterworks sludge as bricks material”, International Journal of Engineering and Applied Sciences, vol. 3, n. ${ }^{\circ}$ 3, pp. 69-79, 2013.

[13] Z. Li, et al., "Experimental investigation of phosphorus adsorption capacity of the waterworks sludges from five cities in China”, Ecological Engineering, vol. 53, pp. 165-172, 2013.

Revista Ingenierías Universidad de Medellín 
[14] H. B. Dharmappa, et al., "Water treatment plant residuals management", Water Science and Technology, vol. 35, n. ${ }^{\circ}$, pp. 45-56, 1997.

[15] J. D. Villegas, et al., "Recuperación de sulfato de aluminio a partir de lodos generados en Plantas de potabilización de agua", Scientia et Technica, vol. 9, pp. 223-228, 2005.

[16] G. R. Xu, et al., "Recycle of Alum recovered from water treatment sludge in chemically enhanced primary treatment”, Journal of Hazardous Materials, vol. 161, n. ${ }^{\circ}$ 2, pp. 663-669, 2009.

[17] A. A. Azim, et al., "Comparison between applying acidification method for alum recovery of different clarifier type", Journal of Applied Sciences Research, vol. 9, n. ${ }^{\circ}$ 8, pp. 4723-4733, 2013.

[18] Y. Q. Zhao, "Settling behaviour of polymer flocculated water-treatment sludge I: analyses of settling curves", Separation and Purification Technology, vol. 35, n. ${ }^{\circ}$ 1, pp. 71-80, 2004.

[19] B. A. Bolto, "Coagulation and flocculation with organic polyelectrolytes", en Interface Science and Technology. Theory and Applications, G. Newcombe y D. Dixon, eds., pp. 63-88, Australia: Elsevier, 2006.

[20] R. B. Sotero-Santos, et al., "Evaluation of water treatment sludges toxicity using the Daphnia bioassay", Water Research, vol. 39, n. ${ }^{\circ}$ 16, pp. 3909-3917, 2005. 